\title{
Mapping evidence of Universal Health Coverage in the world: a protocol for a scoping review
}

Aklilu Endalamaw ( $\square$ yaklilu12@gmail.com )

Bahir Dar University https://orcid.org/0000-0002-9121-6549

Charles F Gilks

The University of Queensland

Fentie Ambaw

Bahir Dar University

Yibeltal Assefa

The University of Queensland

\section{Protocol}

Keywords: Universal Health Coverage, Protocol, scoping review

Posted Date: November 19th, 2021

DOI: https://doi.org/10.21203/rs.3.rs-1082468/v1

License: (9) This work is licensed under a Creative Commons Attribution 4.0 International License. Read Full License 


\section{Abstract \\ Background}

Universal health coverage (UHC) is critical for improving global health and socioeconomic development. Monitoring and evaluating UHC's progress has also been suggested as an additional intervention to improve its road map. Globally, the concept of UHC is being desperately used by health care delivery systems and research institutions using a variety of indicators. More evidence is required to know what is known and the gaps are in the UHC literature that helps to identify research problems. The review will map the available researches of UHC from around the world.

\section{Methods}

The scoping review will be reported using the checklist The Preferred Reporting Items for Systematic Reviews and Meta-analysis Extension for Scoping Reviews (PRISMA-ScR). For studies reporting data on $\mathrm{UHC}$ and its dimensions, systematic searches will be conducted in PubMed, Scopus, Web of Science, Google Scholar, and the United Nations, World Health Organization, and World Bank repositories. To present the review findings, a narrative synthesis will be performed.

\section{Discussion}

UHC is a cornerstone to achieve the health goal of UN's by the end of 2030. The mapping of available literature towards UHC will provide evidence that will help in the designing and implementation of health policy, health research and health care services.

\section{Conclusions}

A protocol for a global scoping review of UHC research is described. This scoping review will add to the body of knowledge about UHC research. The review will identify UHC research questions for which primary research or a systematic review and meta-analysis will be conducted. This is the first review of $\mathrm{UHC}$ to be conducted. This review will prioritize UHC research questions in relation to the sustainable development goals.

\section{Background}

Universal Health Coverage (UHC) is critical to meeting the United Nation's (UN) long-term development goal of 2030. The UHC improves the global population's health and well-being, socio-economic growth and social justice (1) by providing a diverse range of quality and equitable health services without putting clients in financial jeopardy (2). UHC is a topic of international debate; WHO's 2010 report on the path to universal coverage (3), World Bank's 2013 meeting considered UHC as the primary goal (4) and WHO's 
2013 meeting called for UHC research (5). Furthermore, the UN General Assembly in 2012 encouraged countries to follow UHC (6) and the UN's post-2015 goals listed UHC as the predominant goal of financial risk protection, "access to quality essential health-care services and access to safe, effective, quality and affordable essential medicines and vaccines for all” (7).

Sustainable development goal (SDG)-3 target 3.8 is about UHC goal of reaching 80 percent of the population and zero catastrophic expenditure by the end of 2030. The 73rd session of The UN General Assembly also agreed in a political declaration to provide health services to 1 billion more people by 2023 (8). According to the most recent UN high-level meeting on UHC by $2020, \mathrm{UHC}$ is also an urgent priority for ending the current global health crises, including the pandemic COVID-19 (9). Working on UHC is solely for the purpose of reducing inequalities and social exclusion, promoting effective and inclusive governance for health and constructing resilient and sustainable systems (10).

However, having to meet the UHC targets within the expected time frame may be difficult. One of the challenges is a lack of clarity on the measurements used to study UHC, including measuring its progress. Consultancy meetings, international conferences, document reviews, policy inaugurations and stakeholder collaborations have been held to address the challenges that the health system and countries face, with notable examples including the adoption of indicators to measure UHC in in 2017 by the UN General Assembly based on the recommendations of the UN Inter-agency and Expert Group (IAEG) on SDG indicators, which was comprised of national statisticians from 27 countries (11). The UNs Statistical Commission established SDG3.8.1 and 3.8.2 indicators to assess UHC (12). According to the WHO and World Bank Group (13), sixteen tracer indicators were used to track progress toward UHC service coverage in the reproductive, maternal, newborn and child health, infectious diseases, noncommunicable diseases and service capacity and access areas in 2014. The UHC service coverage index is calculated using the direct geometric means of tracer indicators on a scale of 0 to 100 percent. Other than SDG 3.8.2, different approaches are used to measure catastrophic health spending, with estimated benchmark of $10 \%$ or $25 \%$ $(14,15)$. In contrast to WHO, the World Bank and even the 2017 Global Burden of Diseases, Injuries, and Risk Factors Study $(13,16)$, the GBD 2019 (17) proposed 23 UHC effective coverage indicators based on intervention coverage or outcome-based measures such as mortality-to-incidence ratios to approximate access to quality care.

While studies have been conducted on UHC at around the world, with variations on methodology followed and measurement metrics and indicators used, few have been done to identify the type of available evidence with showing the technique how UHC is done across global community. Other resources, according to our searches, include "a synthesis of conceptual literature and global debates" (18) and a scoping review of "implementation research approaches to promoting UHC" (19). Both are not about mapping UHC research done around the world. There is still a gap in assessing the available evidence to support this ambitious agenda. While this is true, the lack of health care services, inequity health care delivery and difficulty in accessing the health care setting are all visible and growing. This review aims to map the available evidence of UHC from around the world. More information is needed to explain what is currently known and the gaps are in the UHC literature in order to determine research priorities. According 
to our knowledge, this will be the world's first scoping review describing the broad, heterogeneous, and complex nature of UHC research.

\section{Methods}

The aims of this scoping review are firstly, to determine the study characteristics, study types and designs and content during the preconception period, overall UHC studies, secondly, to identify gaps in the available literature on $\mathrm{UHC}$ in the world and thirdly, to inform on priority research questions for primary data collection related to $\mathrm{UHC}$ in the world

We will conduct a scoping review of published and grey literature on UHC. The Arksey and O'Malley's (2005) scoping review framework (20), which was expanded on by Levac et al. (2010) methodological enhancement for scoping review projects (21), and the Joanna Briggs Institution framework (22) will be used. The stages in the framework are (1) identification of the research questions, (2) identification of relevant studies, (3) study selection, (4) data charting, (5) collation, summarization and reporting of results and (6) consultation with key stakeholders to communicate and discuss findings from the review. As a guide, the Preferred Reporting Items for Systematic reviews and Meta-analysis extension for Scoping Reviews checklist (PRISMA-ScR) (23) will be used for this scoping review. This scoping review will also employ Population, Concept and Context (PCC) framework to determine the research question's eligibility (Table 1).

\section{Identifying a research question}

The research questions were developed by the authors $A E$ in collaboration with $Y A$ of the research team. The Population, Concept, and Context framework was used by the research teams to determine the eligibility of research questions (Table). According to the framework, the population will include people of any gender at any age, as well as health institutions from around the world, while our concept will be UHC.

\section{Identifying relevant studies}

PubMed, Scopus, Web of Science, Google Scholar, and Google will be used for both published and unpublished literature searches. The relevant keywords listed below will be used to create a comprehensive search strategy (Supplementary file 1). The applicable keywords and/or phrases are Universal, health, "health care", healthcare, "health service", quality, access, coverage, equity, disparity, inequity, equality, inequality, expenditure, and cost. "AND" or "OR" Boolean operators and asterisk search operator will be applied. The UN, World Bank and WHO depository will be accessed for UHC policy and program evaluation studies. To avoid duplication and for citation purposes, references will be collected from each database and stored in EndNote desktop version x7.

Studies done by English will be considered. The search will be restricted to include literature conducted after 2015 because UHC is set as one of health targets in the SDGs in 2015. The searching of articles will 
be conducted between October 20, 2021 to November 12, 2021. We intend to include all studies that adhered to scientific principles, such as systematic data and information system uses and program planning models. Systematic reviews and meta-analysis, randomized trails, observational studies, case studies, evaluation studies and laboratory studies will be included.

Literature that could exclude will be non-English, comment to editor, commentators and brief communications.

\section{Study selection}

AE will create and test study selection forms on a random sample of references derived from search results, with consultation from YA and feedback from CFG and FA. When there is a disagreement, a discussion will be held, and the screening form will be revised.

Following the development of screening forms, literature will be screened using inclusion and exclusion criteria. The title, abstract, and full-text will be reviewed in stages. Titles will be screened after duplicates have been removed using EndNote desktop $x 7$ software and reviewing the title one by one. The literature will then be screened using abstracts. Those passed abstract review will be considered for full-text review. The reasons for the exclusion of studies will be documented. Articles selected for full-text review but with no full-text available will be recorded as well. We will screen full-text articles to include for data charting. Contact will be made with the study's corresponding authors for articles that only include citation and abstract, as well as for any concerns.

\section{Data charting}

To chart the results of the review from full-text literature, a piloted and refined data extraction tool will be developed. Data will be sifted, charted, and sorted based on key issues and themes. The following information will be extracted: author(s), publication or results reported year, data collection year, country of studies represented, WHO geographic category, World Bank income category, study design/type of study, study population, sample size, study question type, studied dimensions, study settings, source of data, measurement of UHC, source of fund, main results and recommendations ( supplementary file 2). AE will collect data, while YA, CFG and FA will review a random sample of articles and provide feedback on the information gathered.

\section{Collation, summarization and report of results}

Literature will be compiled and summarized based on years of publication, country, number of studies included, number of studies by measured dimensions, number of studies by types of studies, countries, WHO and WB categories.

A descriptive analysis will be carried out. We will present the findings in the form of text, graphs and tables. Based on the findings, the data reporting scheme will be adjusted as needed. 
There was no involvement of patients and the public to develop the protocol. Databases that require an institutional license will be accessed through the library at The University of Queensland Australia. The findings will be presented at conferences at The University of Queensland in Australia, Bahir Dar University in Ethiopia, and other locations around the world. Finally, the review's findings will be published in a peer-reviewed journal and disseminated to global communities.

\section{Discussion}

The proposed scoping review study aims to map existing evidences for the overall UHC and its dimensions, namely effective service coverage, financial coverage, population coverage, quality and equity of services in the context of SDG 3.8 target. UHC is a cornerstone to achieve the health goal of UN's by the end of 2030. The mapping of available literature towards UHC will provide evidence that will help in the designing and implementation of health policy, health research and health care services.

\section{Conclusions}

A protocol for a global scoping review of UHC research is described. This scoping review will add to the body of knowledge about UHC research. The review will identify UHC research questions for which primary research or a systematic review and meta-analysis will be conducted. This is the first review of $\mathrm{UHC}$ to be conducted. This review will prioritize UHC research questions in relation to the SDGs.

\section{Abbreviations}

GBD-Global Burden Disease; SDGs-Sustainable Development Goals; UN-United Nations; UHC-Universal Health Coverage; WB-World Bank; WHO-World Health Organization

\section{Declarations}

Ethics approval and consent to participate: Not applicable

Consent for publication: Not applicable

Availability of data and materials: Not applicable

Competing interests: The authors declare that they have no competing interests.

Funding: No fund has received to write the protocol

Acknowledgements: Not applicable

\section{References}


1. Tangcharoensathien V, Mills A, Palu T. Accelerating health equity: the key role of universal health coverage in the Sustainable Development Goals. Bmc Medicine. 2015;13.

2. Thomson S, Cylus J, Evetovits T, editors. How you measure matters: monitoring financial protection to generate policy-relevant evidence for universal health coverage-lessons from Europe. Organized session 9 July 2017. IHEA 12th World Congress in Health Economics; 2017.

3. World Health Organization. The world health report: health systems financing: the path to universal coverage: executive summary. World Health Organization, 2010.

4. Kim JY. Speech by World Bank Group President Jim Yong Kim at the Government of Japan-World Bank Conference on universal health coverage. The World Bank, 2013.

5. World Health Organization. Research for universal health coverage: World health report 20132013 [cited 202115 August]. Available from: https://www.who.int/publications/i/item/9789240690837.

6. United Nations. Resolution adopted by the General Assembly on 12 December 20122013 [cited 202123 October]. Available from: https://undocs.org/A/RES/67/81.

7. United Nations. Resolution adopted by the General Assembly on 25 September 20152015 [cited 202123 October]. Available from: https://undocs.org/A/RES/70/1.

8. United Nations. Universal Health Coverage: Moving Together to Build a Healthier World New York2019 [cited 202124 October]. Available from: https://www.un.org/pga/73/event/universal-healthcoverage/.

9. uhc 2030. State of commitment to universal health coverage: synthesis, 20202020 [cited 2021 October 2]. Available from: https://www.uhc2030.org/blog-news-events/uhc2030-news/state-ofcommitment-to-universal-health-coverage-synthesis-2020-555434/.

10. UNDP. Universal health coverage for sustainable development - issue brief 2019 [cited 2021 October 2]. Available from: https://www.undp.org/publications/universal-health-coverage-sustainabledevelopment-issue-brief.

11. UN. General Assembly (71st sess. : 2016-2017). Work of the Statistical Commission pertaining to the 2030 Agenda for Sustainable Development : resolution / adopted by the General Assembly New York2017 [cited 2021 October 23]. Available from: https://digitallibrary.un.org/record/1291226?ln=en.

12. United Nations. General Assembly, G.A. Res. 71/313, UN Doc. A/RES/71/313 (2017).

13. World Health Organization and World Bank Group. Monitoring progress towards universal health coverage at country and global levels: framework, measures and targets. World Health Organization, 2014. 
14. World Health Organization and The World Bank Group. Tracking Universal Health Coverage: 2017 Global Monitoring Report. Switzerland: 2017.

15. Wagstaff A, Cotlear D, Eozenou PHV, Buisman LR. Measuring progress towards universal health coverage: with an application to 24 developing countries. Oxford Review of Economic Policy. 2016;32(1):147-89.

16. Collaborators GS. Measuring progress from 1990 to 2017 and projecting attainment to 2030 of the health-related Sustainable Development Goals for 195 countries and territories: a systematic analysis for the Global Burden of Disease Study 2017. Lancet (London, England). 2018;392(10159):2091.

17. Lozano R, Fullman N, Mumford JE, Knight M, Barthelemy CM, Abbafati C, et al. Measuring universal health coverage based on an index of effective coverage of health services in 204 countries and territories, 1990-2019: a systematic analysis for the Global Burden of Disease Study 2019. The Lancet. 2020;396(10258):1250-84.

18. Abiiro GA, De Allegri M. Universal health coverage from multiple perspectives: a synthesis of conceptual literature and global debates. Bmc International Health and Human Rights. 2015;15.

19. Nnaji CA. Implementation research approaches to promoting universal health coverage in Africa: a scoping review. The International journal of health planning and management. 2021;21(1):414.

20. Arksey H, O'Malley L. Scoping studies: towards a methodological framework. International journal of social research methodology. 2005;8(1):19-32.

21. Levac D, Colquhoun H, O'Brien KK. Scoping studies: advancing the methodology. Implementation science. 2010;5(1):1-9.

22. Peters MDJ, Marnie C, Tricco AC, Pollock D, Munn Z, Alexander L, et al. Updated methodological guidance for the conduct of scoping reviews. JBI evidence implementation. 2021;19(1):3-10.

23. Tricco AC, Lillie E, Zarin W, O'Brien KK, Colquhoun H, Levac D, et al. PRISMA Extension for Scoping Reviews (PRISMA-ScR): Checklist and Explanation. Ann Intern Med. 2018;169(7):467-73.

\section{Tables}

Table: population, concept and context framework

\begin{tabular}{|ll|}
\hline Criteria & \\
\hline Population & All population in the world \\
\hline Concept & UHC \\
\hline Context & World \\
\hline
\end{tabular}




\section{Supplementary Files}

This is a list of supplementary files associated with this preprint. Click to download.

- Supplementaryfile1.docx

- Supplementaryfile2.docx 